\title{
Contents, Vol. 93, 1937
}

Inhaltsverzeichnis.

Eigenarbeiten. geite

Cassuto, N., Die durch die bipolaren Bazillen verursachten

Bindehautaffektionen 57

Fieischanderl, A., Zur Kenntnis metastatischer Lidtumoren 31 Friede, R., Ueber Salbenfüilung des Bindehautsackes . . 49 Gifford, S. R., Zur Frage des als Parinaudsche Konjunkti-

vitis beschriebenen Krankheitsbildes . . . . 52

Erwiderung zum vorstehenden Artikel. Von A. Piiiat-

Graz 55

Grósz, St. von, Die Cutireaktion der Lues in ophthalmologi-

scher Beleuchtung . . 186

Haiismann, Gertrud, Bericht über die Kurzwellentherapie an

der I. Universitäts-Augenklinik in Wien .... 213 Hoehne, H., Zur Stauungspapille bei Hypotonie des Auges . 133 Kreibig, W., Ueber Karzinommetastasen im Auge. (Hierzu

Tafel I) .... @ 278

Lindner, K., Ueber die Anlegung einer Glaskörperfistel . . 117 Lisch, K., Ueber Beteiligung der Augen, insbesondere das

Vorkommen von Irisknötchen bei der Neurofibromatose

(Recklinghausen) 137

Ohm, Ausziehung des Altersstars mit dem Häkchen . . 65 Pantasatos, G., Ueber Nekrosen in sympathisierenden Infil-

traten und über Periphlebitis retinalis sympathicans . 293 Pillat, A., siehe unter Gifford.

Radnót, Magda, Ueber den Unterschied zwischen Periphlebitis retinalis und Thrombangitis

obliterans retinae . 35 Rollin, Jean L., Zur Frage der Entstehung des Plasmoms (Pa-

scheff) 181

Safer, K., Netzhautabhebungen mit Löchern am hintern Au-

genpol, geheilt durch diathermische Stichelung . . 261 Salzmann, M., Die Iridectomia ab externo. (Statistik u. Er-

gebnisse.) 1

Teegler, Charlotte, Ueber partiellen Arcus lipoides bei lim-

busnahen tumorösen Prozessen der Konjunktiva . 197 Tertsch, R., Drei Fälle von Abdrängung

der Glaskörpergrenz-

membran 121

IV

Inhaltsverzeichnis

Aus der Praxis für die Praxis. geite

Handmann, Totale inversio (recessio) Iridis durch spate

Nachblutung nach Kontusion

222

Kraupa, E, Iridotomie bei der Staroperation .... 
Berichte über die ophthalmologische Literatur.

Linse, Glaskörper, Orbita, Schädel, Nebenhöhlen. (Berichtsjahr vom 1. Janua $\Gamma$ bis 31. Dezember 1936.) Von Dr.

Annemaríe JVeí > e-Marburg a. d. L. . . . Bewegungsapparat des Auges. Von Dr. G. JanckeKöln . Nystagmus (BerichtsjahГ 1936). Von Dr. A. Kestenbaum-

Wien

Sehbahn, Auge und Nervenkrankheiten (Berichtsjahr 1936).

Von Werner Kyríeleis-H'ãmburg .

144

225

300

Gesellschaftsberichte.

Bericht über die Tagung der Gesellschaft der Augenärzte Groß-Ham-

burgs am 15. Januar $1937 \quad 105$

Bericht über die 43. Tagung der Vereinigung mitteldeutscher Augenärzte, 5. und 6. Juni 1937 349

Bericht über die Tagung der nordwestdeutschen augenärztlichen Gesellschaft am 26. Juni 1937 in Rostock 240

Verein Rheinisch-Westfälíscher Augenärzte:

70. Versammlung vom 5. November 1936 in Düsseldorf 103

Sitzung vom 18. April 1937 in Düsseldorf . . $\quad 345$

Schwcizerische Ophthalmologische Gesellschaft. 30. Jahresversammlung

am 5. und 6. Juni 1937 in Basel 333

Deutsche ophthalmologische Gesellschaft in der tschechoslowakischen

Republik. Versammlung am 5. und 6. Dezember 1936 in Prag . 158

Ophthalmologische Gesellschaft in Wien:

Sitzung vom 15. März $1937 \quad 96$

Sitzung vom 24. Mai $1937 \quad 235$

Gesellschafl der Augenärzte von Zurich. Versammlung vom 8. Dez. 193694

Diagnose und Therapie . Buchbesprechungen . Die Arlt-Feier in Obergraupen. Personalien ...

Dozent Dr. Richard Kramer † Tagesnachrichten Saehregister zn Band 93 . Namensregister zu

Band 93

Von K. Lindner

W. Wien

113, 176, 254, 363 180, 259179 259, 367367180368372 